Our Nature (2009) 7:100-109

\title{
Avifaunal Diversity of Gudavi Bird Sanctuary, Sorab, Shimoga, Karnataka
}

\author{
G.Y. Dayananda \\ Department of Applied Zoology, Biosciences complex, Jnana Sahyadri, Kuvempu University, Shankaraghatta- \\ 577 451, Shimoga, Karnataka, India \\ E-mail:dayananda_gy@yahoo.com
}

Received: 21.01.2009, Accepted: 09.04.2009

\begin{abstract}
Two hundred and seventeen species of birds belonging to 48 families were recorded in the three year study at the Gudavi sanctuary, Sorab, Shimoga district, Karnataka. Most of the families were represented by less than thirteen species except for Muscicapidae (35 species). These include 160 residents, 46 resident migratory and 11 migratory birds. 54 species of water dependent birds were recorded from the wetlands of the sanctuary. Among the birds recorded in this study, about $45.62 \%$ are insectivores and other dominating types include piscivores $(12.44 \%)$, omnivores $(11.98 \%)$, frugivores $(9.67 \%)$, grainivores $(8.75 \%)$, carnivores $(7.83 \%)$ and nectarivores $(3.68 \%)$ respectively.
\end{abstract}

Key words: Avifauna, Biodiversity, Gudavi Bird Sanctuary, Sorab

\section{Introduction}

The birds have always fascinated man for their exquisite colouration. They have their functional role in the ecosystem as potential pollinators and scavengers, indeed rightly called bioindicators. India being a megadiversity centre, harbours more than 1200 species of birds which amounts to $13 \%$ of the bird species of the world (Ali and Ripley, 1983). Recently with the increased consciousness for biodiversity census and monitoring, many new species were added to the list.

The present study is focused not only on preparing the checklist of birds, but also to find out their occurrence status as well as to create awareness for their conservation. In addition, the study aims at providing the basic information of the avifauna for further studies related to biodiversity of wildlife sanctuary. This is the only the large birds area in Shimoga district, where more than
12,000 wetland birds congregate every year. Hence detailed study on avifauna of Gudavi bird sanctuary (GBS) is undertaken.

\section{Methodology \\ Study area}

Gudavi bird sanctuary is one of the five bird sanctuaries of Karnataka, with notification, AHFF-262-fwl 86 on 10:07:1989 (Figure 1). It is located at $13 \mathrm{~km}$ away from Sorab city and $0.5 \mathrm{~km}$ away from Gudavi village. This sanctuary occupies the water-spread area of about 33 ha, in rainy season, out of the total 73.68 ha. Remaining area is moist deciduous forest interspread with grassy patches. The sanctuary lies between Lat. $14^{\circ} 25^{\prime} 59^{\prime \prime}-14^{\circ} 26^{\prime} 41^{\prime \prime} \mathrm{N}$ and Long. $75^{\circ} 643^{\prime \prime}-75^{\circ} 25^{\prime} 28^{\prime \prime} \mathrm{E}$.

There are two wetlands adjoining each in the sanctuary (Vaddekere and Gudavi pond). The rainfed water from agriculture field enters into Vaddekere and the outflow 
of water from Vaddekere enters into Gudavi pond, from their in is used for irrigation at down stream croplands. The Vaddekere and Gudavi pond are separated by a common bund. All breeding birds breed at Vaddekere during breeding season and roost on trees of Gudavi pond.

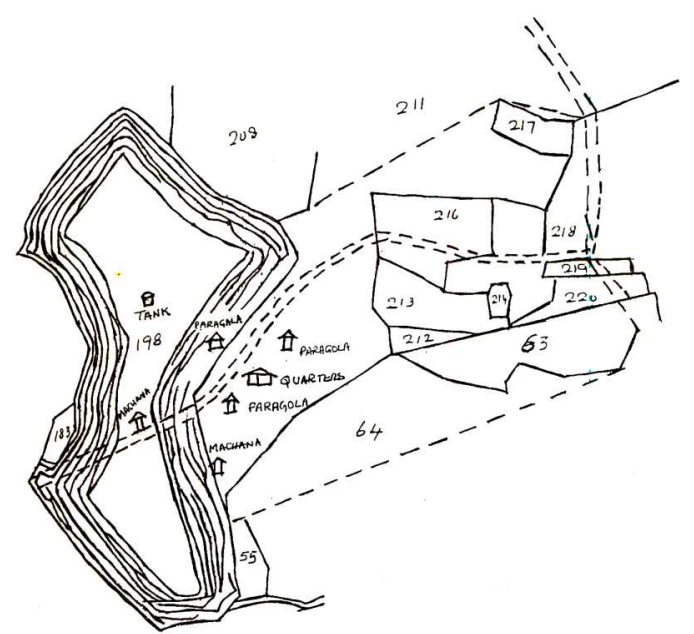

Figure 1. Gudavi Bird Sanctuary

\section{Vegetation}

The area surrounding the wetland is covered with dense moist deciduous forest, and due to intensive protection efforts by the Forest Department, the area has maintained greenery. Apart from this, Gudavi sanctuary embodies diversified vegetation that favors the migratory birds to take shelter and provides the space to construct their nests for breeding. The vegetation water spread area of GBS comprised of marshy plants and microphytes. The trees and shrubs partially submerged and provide suitable nesting sites for birds. These nesting sites also provide the highest security from the predators.

\section{Methods}

The checklist of birds at the sanctuary was made by sighting the birds with $8 \times 50$ binoculars. Appropriate field guides (Ali, 1996; Sonobe and Usui, 1993) were used for the purpose. Based on regularly updated checklist the detailed census of birds was conducted by direct count method (Colin et $a l .$, 1993). The study was conducted at monthly intervals from 2001 to 2003 by scanning the sanctuary by walking. The morning hours, 0630 to $1000 \mathrm{am}$ and evening 0330 to $0700 \mathrm{pm}$, were utilized for the purpose. The monthly totals of individual species and the total population was enumerated from three vantage points on the countour of each pond.

\section{Results and discussion}

The present study on the diversity of birds inhabiting the Vaddekere and Gudavi pond suggests that the physico-chemical and biological parameters of their habitat are the major regulating forces of their population density (Dayananda, 2005). The avifauna of GBS belonged to 16 orders. Out of these members of Ciconiformes, Paliconiformes and Passeriformes contributed maximum to the avifauna of the ponds throughout the year. The members of order Anseriformes and Charadriformes are migratory species who utilize the ponds for foraging during winter months. However, the local migrants are widespread throughout the year and to adjacent wetlands, moving to and fro utilizing the best resources available to them. Raghunatha et al. (1992) recorded 191 bird species during his preliminary report. Presently the total number of species recorded in this sanctuary is 218 .

The checklist of birds sighted at the sanctuary during the three-year study, the residential status and abundance is provided in Table 1. Of the 218 species of birds 


\section{G.Y. Dayananda / Our Nature (2009) 7: 100-109}

sighted at the sanctuary, a large number of terrestrial birds (163 species) constituted $75.11 \%$ whereas $24.88 \%$ was constituted by a relatively small number of aquatic birds consisting of 54 species. In terms of species strength of families represented Muscicapidae was the largest with 35 species. In the present study, the bird species are classified into six groups based on their food preference. Among these bird species, 99 were Insectivorous, 27 piscivorous, 26 omnivorous, 21 frugivorous, 20 grainivorous, 17 carnivorous and 8 nectarivorous birds respectively.

\section{Acknowledgements}

We are thankful to Mr. G.A.N. Karkikar, Deputy Conservator of Forest, Wild Life Wing, Shimoga and Mr. Ramappa, Forest watcher, Gudavi Bird Sanctuary for their necessary guidance and help during the study period.

\section{References}

Ali, S. 1996. The book of Indian birds. $12^{\text {th }}$ edition, Bombay Natural History Society, Oxford University Press, Bombay. 354 p.

Ali, S. and S.D. Ripley 1983. Hand book of birds of India and Pakistan. Oxford University Press, Delhi. pp. 110-112.

Kumar, A., J.P. Sati and P.C. Tak 2003. Checklist of Indian water birds. ENVIS Newsletter: Avian Ecology and Inland Wetlands. Bombay Natural History Society. Buceros 8(1): 1-30.

Colin, J., D. Bibby, Neil, Burgess, A. David and Hill 1993. Text book of birds census techniques. Academic Press Ltd., London. pp. 24-28.

Dayananda, G.Y. 2005. Ornitho-ecological studies of Gudavi Bird Sanctuary, Sorab, Karnataka., Kuvempu University, Karnataka. 1-146. (Ph.D. Thesis)

Manakadan, R. and A. Pittie 2001. Standardized common and scientific names of the Birds of the Indian subcontinent. Buceros 6(1): 1-37.

Pittie, A. and A. Robertson 1993. Nomenclature of birds of the Indian subcontinent-A review of some changes taking place. Ornithological Society of India, Bangalore.

Raghunatha, S., Subramanya, Shyamal, Lokesha and R. Vasudeva 1992. A preliminary survey of Gudavi bird Sanctuary. My Forest 28(3): 265-274.

Sonobe, K. and S. Usui 1993. A field guide to the water birds of Asia. Wild Bird Society of Japan, Tokyo.

Table 1. A systematic list of birds with their status and food habit in Gudavi Bird Sanctuary during the study period (2001, 2002 and 2003)

\begin{tabular}{|c|c|c|c|c|c|c|}
\hline $\mathrm{SN}$ & Syn No & Common name & Scientific Name & $\mathrm{RS}^{1}$ & $\mathrm{AS}^{2}$ & $\mathrm{FH}^{3}$ \\
\hline \multicolumn{7}{|c|}{ 1. Podicipedidae } \\
\hline 1. & 5 & Little Grebe & Tachybaptus ruficollis & $\mathrm{R}$ & V Com & $\mathrm{P}$ \\
\hline \multicolumn{7}{|c|}{ 2. Phalacrocoracedae } \\
\hline 2. & 26 & Great Cormorant & Phalacrocorax carbo & $\mathrm{RM}$ & Com & $\mathrm{P}$ \\
\hline 3. & 27 & Indian Shag & Phalacrocorax fuscicollis & $\mathrm{RM}$ & Com & $\mathrm{P}$ \\
\hline 4. & 28 & Little Cormorant & Phalacrocorax niger & $\mathrm{RM}$ & L Com & $\mathrm{P}$ \\
\hline \multicolumn{7}{|c|}{ 3. Anhingidae } \\
\hline 5. & 29 & Darter or Snake bird & Anhing a melamogaster & $\mathrm{RM}$ & L Com & $\mathrm{P}$ \\
\hline \multicolumn{7}{|c|}{ 4. Ardeidae } \\
\hline 6. & 36 & Grey Heron & Ardea cinerea & $\mathrm{RM}$ & L Com & $\mathrm{P}$ \\
\hline 7. & 37 & Purple Heron & Ardea purpurea & $\mathrm{RM}$ & L Com & $\mathrm{P}$ \\
\hline 8. & 38 & Little Green Heron & Butorides striatus & $\mathrm{RM}$ & $\mathrm{Ra}$ & $\mathrm{P}$ \\
\hline 9. & 42 & Pond Heron & Ardeola grayii & $\mathrm{R}$ & L Com & $\mathrm{P}$ \\
\hline 10. & 44 & Cattle Egret & Bubulcus ibis & $\mathrm{RM}$ & Com & $\mathrm{P}$ \\
\hline
\end{tabular}


G.Y. Dayananda / Our Nature (2009) 7: 100-109

\begin{tabular}{|c|c|c|c|c|c|c|}
\hline 11. & 46 & Large Egret & Casmerodius albus & $\mathrm{RM}$ & Com & $\mathrm{P}$ \\
\hline 12. & 47 & Smaller Egret & Mesophoyx intermedia & $\mathrm{RM}$ & L Com & $\mathrm{P}$ \\
\hline 13. & 49 & Little Egret & Egretta garzetta & $\mathrm{R}$ & L Com & $\mathrm{P}$ \\
\hline 14. & 52 & Night Heron & Nycticorax nycticorax & $\mathrm{R}$ & L Com & $\mathrm{P}$ \\
\hline 15. & 56 & Chestnut Bittem & Ixobrychus cinnamoneus & $\mathrm{RM}$ & L Com & $\mathrm{P}$ \\
\hline \multicolumn{7}{|c|}{ 5. Ciconiidae } \\
\hline 16. & 60 & Painted Stork & Mycteria leucocephala & $\mathrm{RM}$ & L Com & $\mathrm{P}$ \\
\hline 17. & 61 & Openbill Stork & Anastomus oscitans & $\mathrm{R}$ & L Com & $\mathrm{P}$ \\
\hline \multicolumn{7}{|c|}{ 6. Threskiomithidae } \\
\hline 18. & 69 & Black-headed Ibis & Threskiornis melanocephalus & $\mathrm{R}$ & L Com & $\mathrm{P}$ \\
\hline 19. & 70 & Black Ibis & Pseudibis papillosa & $\mathrm{R}$ & Un Com & $\mathrm{P}$ \\
\hline 20. & 71 & Glossy Ibis & Plegadis falcinellus & $\mathrm{RM}$ & Un Com & $\mathrm{P}$ \\
\hline 21. & 72 & Spoonbill & Platalea leucorodia & $\mathrm{RM}$ & L Com & $\mathrm{P}$ \\
\hline \multicolumn{7}{|c|}{ 7. Anatidae } \\
\hline 22. & 88 & Lesser whistling Teal & Dendrocygna javanica & $\mathrm{R}$ & L Com & $\mathrm{O}$ \\
\hline 23. & 93 & Pintail & Anus acuta & M & Com & $\mathrm{O}$ \\
\hline 24. & 94 & Common Teal & Anas crecca & $\mathrm{RM}$ & V Com & $\mathrm{O}$ \\
\hline 25. & 97 & Spot-billed Duck & Anas poecilorhyncha & $\mathrm{RM}$ & Com & $\mathrm{O}$ \\
\hline 26. & 104 & Garganey & Anas querquedula & M & V Com & $\mathrm{O}$ \\
\hline 27. & 105 & Shoveller & Anas clypeata & M & Com & $\mathrm{O}$ \\
\hline 28. & 114 & Cotton Teal & Nettapus coromandelianus & $\mathrm{R}$ & L Com & $\mathrm{O}$ \\
\hline 29. & 115 & Nakta or Comb Duck & Sarkidiornis melanotos & $\mathrm{R}$ & Un Com & $\mathrm{O}$ \\
\hline \multicolumn{7}{|c|}{ 8. Accipitridae } \\
\hline 30. & 130 & Crested Honey-Buzzard & Pernis ptilorhyncus & $\mathrm{R}$ & L Com & $\mathrm{C}$ \\
\hline 31. & 133 & Common Pariahkite & Milvus migrans & $\mathrm{R}$ & Com & $\mathrm{C}$ \\
\hline 32. & 135 & Brahminy kite & Haliasturindus & $\mathrm{R}$ & L Com & $\mathrm{C}$ \\
\hline 33. & 139 & Shikra & Accipiter badius & $\mathrm{RM}$ & Com & $\mathrm{C}$ \\
\hline 34. & 147 & Sparrow Hawk & Accipiter nisus & $\mathrm{R}$ & L Com & $\mathrm{C}$ \\
\hline 35. & 151 & Besra Sparrow Hawk & Accipiter virgatus & $\mathrm{R}$ & L Com & $\mathrm{C}$ \\
\hline 36. & 168 & Tawny Eagle & Aquila rapax & $\mathrm{RM}$ & L Com & $\mathrm{C}$ \\
\hline 37. & 170 & Greater spotted Eagle & Aquila clanga & $\mathrm{RM}$ & $\mathrm{Ra}$ & $\mathrm{C}$ \\
\hline 38. & 174 & Ring tailed fishing Eagle & Haliaeetus leucoryphus & M & $\mathrm{Ra}$ & $\mathrm{C}$ \\
\hline 39. & 190 & Pale Harrier & Circus macrourus & $\mathrm{RM}$ & L Com & $\mathrm{C}$ \\
\hline 40 & 192 & Paid Harrier & Circus melanoleucos & M & $\mathrm{Ra}$ & $\mathrm{C}$ \\
\hline 41. & 193 & Marsh Harrier & Circus aeruginosus & $\mathrm{R}$ & L Com & $\mathrm{C}$ \\
\hline 42. & 222 & Common Kestrel & Falco tinnunculus & $\mathrm{RM}$ & L Com & $\mathrm{C}$ \\
\hline \multicolumn{7}{|c|}{ 9. Phasianidae } \\
\hline 43. & 244 & Grey Partridge & Francolinus pondicerianus & $\mathrm{R}$ & Com & $\mathrm{O}$ \\
\hline 44. & 250 & Jungle bush Quill & Perdicula asiatica & $\mathrm{R}$ & Com & $\mathrm{O}$ \\
\hline 45 & 275 & Red Spurfowl & Galloperdix spadicea & $\mathrm{R}$ & L Com & $\mathrm{F}, \mathrm{G}, \mathrm{I}$ \\
\hline 46. & 301 & Grey Jungle fowl & Gallus sonneratii & $\mathrm{R}$ & Com & $\mathrm{G}, \mathrm{I}$ \\
\hline 47. & 311 & Common Peafowl & Pavo cristatus & $\mathrm{R}$ & Com & $\mathrm{O}$ \\
\hline
\end{tabular}


G.Y. Dayananda / Our Nature (2009) 7: 100-109

\begin{tabular}{|c|c|c|c|c|c|c|}
\hline \multicolumn{7}{|c|}{ 10. Turnicidae } \\
\hline 48. & 318 & Common Bustard Quail & Turnix suscitator & $\mathrm{R}$ & L Com & $\mathrm{O}$ \\
\hline \multicolumn{7}{|c|}{ 11. Rallidae } \\
\hline 49. & 329 & $\begin{array}{l}\text { Indian Blue-breasted Banded } \\
\text { Rail }\end{array}$ & Rallus striatus & $\mathrm{RM}$ & Un Com & $\mathrm{O}$ \\
\hline 50 . & 332 & Slaty-legged Banded Crake & Rallina eurizonoides & $\mathrm{R}$ & Un Com & I \\
\hline 51. & 342 & Brown Crake & Amaurornis akool & $\mathrm{R}$ & Com & I \\
\hline 52. & 343 & White breasted Water hen & Amaurornis phoenicurus & $\mathrm{R}$ & Com & $\mathrm{I}, \mathrm{G}$ \\
\hline 53. & 346 & Water Cock & Gallicrex cinerea & $\mathrm{RM}$ & L Com & I \\
\hline 54. & 347 & Indian Moorhen & Gallinula chloropus & $\mathrm{R}$ & Com & $\mathrm{O}$ \\
\hline 55. & 348 & Puple Moomen & Porphyrio porphyrio & $\mathrm{RM}$ & L Com & $\mathrm{O}$ \\
\hline 56. & 350 & Coot & Fulica atra & $\mathrm{R}$ & V Com & $\mathrm{O}$ \\
\hline \multicolumn{7}{|c|}{ 12. Jacanidae } \\
\hline 57. & 358 & Pheas ant tailed Jacana & Hydrophasianus chirurgus & $\mathrm{R}$ & Un Com & $\mathrm{I}, \mathrm{G}$ \\
\hline 58. & 359 & Bronze winged Jacana & Metopidicus indicus & $\mathrm{R}$ & L Com & $\mathrm{I}, \mathrm{G}$ \\
\hline \multicolumn{7}{|c|}{ 13. Charadriidae } \\
\hline 59. & 366 & Red-wattled Lapwing & Vanellus indicus & $\mathrm{R}$ & Com & I \\
\hline 60. & 370 & Yellow-wattled Lapwing & Vanellus malabaricus & M & L Com & I \\
\hline 61. & 371 & Grey Plover & Pluvialis squatarola & M & Un Com & I \\
\hline 62. & 373 & Golden Plover & Pluvialis dominica & $\mathrm{RM}$ & $\mathrm{Va}$ & I \\
\hline 63. & 380 & Little ringed Plover & Charadrius dubius & M & Com & I \\
\hline 64. & 395 & Marsh Sandpiper & Tringa stagnatilis & M & L Com & I \\
\hline 65. & 398 & Spotted Sandpiper & Tring a glareola & $\mathrm{RM}$ & L Com & $\mathrm{I}$ \\
\hline \multicolumn{7}{|c|}{ 14. Scolopacidae } \\
\hline 66. & 409 & Common or Fantail Snipe & Gallinago gallinago & $\mathrm{R}$ & Com & $\mathrm{I}$ \\
\hline \multicolumn{7}{|c|}{ 15. Recurvirostridae } \\
\hline 67. & 430 & Black-winged Stilt & Himantopus himantopus & $\mathrm{R}$ & Com & $\mathrm{I}$ \\
\hline \multicolumn{7}{|c|}{ 16. Lanidae } \\
\hline 68. & 463 & Indian River Tern & Sterna aurantia & $\mathrm{RM}$ & L Com & $\mathrm{P}$ \\
\hline 69. & 464 & Common Tern & Sterna hirundo & $\mathrm{R}$ & L Com & $\mathrm{P}$ \\
\hline \multicolumn{7}{|c|}{ 17. Culumbidae } \\
\hline 70 . & 496 & Grey fronted Green Pigeon & Treron pompadora & $\mathrm{R}$ & L Com & $\mathrm{G}, \mathrm{F}$ \\
\hline 71 & 503 & Common Green Pigeon & Treron phoenicoptera & $\mathrm{R}$ & Com & $\mathrm{G}, \mathrm{F}$ \\
\hline 72. & 510 & Green Imperial Pigeon & Ducula badia & $\mathrm{R}$ & L Com & $\mathrm{G}, \mathrm{F}$ \\
\hline 73. & 516 & Blue Rock Pigeon & Columba livia & $\mathrm{R}$ & V Com & $\mathrm{G}, \mathrm{F}$ \\
\hline 74. & 537 & Spotted Dove & Streptopelia chinen sis & $\mathrm{R}$ & V Com & $\mathrm{G}, \mathrm{F}$ \\
\hline \multicolumn{7}{|c|}{ 18. Psittacidae } \\
\hline 75 . & 550 & Rose ringed $\mathrm{P}$ arakeet & Psittacula krameri & $\mathrm{R}$ & V Com & $\mathrm{F}$ \\
\hline 76. & 558 & Blossomheaded Parakeet & Psittacula cyanocephala & $\mathrm{RM}$ & L Com & $\mathrm{F}$ \\
\hline 77. & 566 & Lorikeet & Loriculus vernalis & $\mathrm{RM}$ & L Com & $\mathrm{F}$ \\
\hline \multicolumn{7}{|c|}{ 19. Cuculidae } \\
\hline 78. & 571 & Pied crested Cuckoo & Clamatorjacobinus & $\mathrm{R}$ & L Com & $\mathrm{F}, \mathrm{I}$ \\
\hline 79. & 573 & Common hawk Cuckoo & Cuculus varius & $\mathrm{R}$ & L Com & $\mathrm{F}, \mathrm{I}$ \\
\hline 80 . & 576 & Indian Cuckoo & Cuculus micropterus & $\mathrm{R}$ & L Com & $\mathrm{F}, \mathrm{I}$ \\
\hline
\end{tabular}


G.Y. Dayananda / Our Nature (2009) 7: 100-109

\begin{tabular}{|c|c|c|c|c|c|c|}
\hline 81. & 590 & Koel & Eudynamys scolopacea & $\mathrm{R}$ & V Com & $\mathrm{F}, \mathrm{I}$ \\
\hline 82. & 593 & Large Greenbilled Malkoha & Rhopodytes tristis & $\mathrm{R}$ & L Com & $\mathrm{F}, \mathrm{I}$ \\
\hline 83. & 595 & Small Greenbilled Malkoha & Rhopodytes viridirostris & $\mathrm{R}$ & L Com & $\mathrm{F}, \mathrm{I}$ \\
\hline 84. & 600 & Coucal or Crow-Pheasant & Centropus sinensis & $\mathrm{R}$ & V Com & I \\
\hline 85. & 605 & Lesser Coucal & Centropus bengalensis & $\mathrm{R}$ & V Com & I \\
\hline \multicolumn{7}{|c|}{ 20. Strigidae } \\
\hline 86. & 636 & Barred Jungle Owlet & Glaucidium radiatum & $\mathrm{R}$ & L Com & $\mathrm{C}$ \\
\hline 87. & 652 & Spotted Owlet & Athene brama & $\mathrm{R}$ & Com & $\mathrm{C}$ \\
\hline 88. & 657 & Mottled wood Owl & Strix ocellata & $\mathrm{R}$ & L Com & $\mathrm{C}$ \\
\hline 89. & 659 & Brown wood Owl & Strix leptogrammica & $\mathrm{RM}$ & L Com & $\mathrm{C}$ \\
\hline \multicolumn{7}{|c|}{ 21. Apodidae } \\
\hline 90. & 685 & Indian edible nest Swiftlet & Collocalia unicolor & $\mathrm{R}$ & Com & $\mathrm{I}$ \\
\hline 91. & 703 & House Swift & Apus affinis & $\mathrm{RM}$ & Com & $\mathrm{I}$ \\
\hline \multicolumn{7}{|c|}{ 22. Alcedinidae } \\
\hline 92. & 719 & Pied Kingfisher & Ceryle rudis & $\mathrm{R}$ & Com & $\mathrm{P}$ \\
\hline 93. & 722 & Small blue King fisher & Alcedo at this & $\mathrm{R}$ & Com & $\mathrm{P}$ \\
\hline 94. & 730 & Stork-billed Kingfisher & Pelargopsis capensis & $\mathrm{R}$ & L Com & $\mathrm{P}$ \\
\hline 95. & 735 & White-breasted Kingfisher & Halcyon smyrnensis & $\mathrm{R}$ & Com & $\mathrm{P}$ \\
\hline \multicolumn{7}{|c|}{ 23. Meropidae } \\
\hline 96. & 744 & Chestnut headed Bee-ater & Merops leschenaultia & $\mathrm{RM}$ & L Com & I \\
\hline 97. & 750 & Small green Bee-eater & Merops orientalis & $\mathrm{R}$ & L Com & I \\
\hline 98. & 753 & Blue bearded Bee-eater & Nyctyornis athertoni & $\mathrm{R}$ & L Com & I \\
\hline \multicolumn{7}{|c|}{ 24. Coraciidae } \\
\hline 99. & 755 & Roller or Blue jay & Coracias benghalensis & $\mathrm{R}$ & Com & $\mathrm{I}$ \\
\hline \multicolumn{7}{|c|}{ 25. Upupidae } \\
\hline 100. & 765 & Hoopoe & Upupa epops & $\mathrm{R}$ & V Com & I \\
\hline \multicolumn{7}{|c|}{ 26. Bucerotidae } \\
\hline 101. & 767 & Common grey Hombill & Tockus birostris & $\mathrm{R}$ & Com & $\mathrm{F}$ \\
\hline 102. & 768 & Malabar Grey Hornbill & Tockus griseus & $\mathrm{R}$ & Com & $\mathrm{F}$ \\
\hline 103. & 775 & Malabar pied Hombill & Anthracoceros coronatus & $\mathrm{R}$ & Com & $\mathrm{F}$ \\
\hline \multicolumn{7}{|c|}{ 27. Megal aimidae } \\
\hline 104. & 782 & Large green Barbet & Megalaima zeylanica & $\mathrm{R}$ & Com & $\mathrm{F}$ \\
\hline 105. & 783 & Lineated Barbet & Megalaima lineate & $\mathrm{R}$ & L Com & $\mathrm{F}$ \\
\hline 106. & 785 & Small green Barbet & Megalaima viridis & $\mathrm{R}$ & Com & $\mathrm{F}$ \\
\hline 107. & 790 & Crimson throated Barbet & Megalaima rubricapilla & $\mathrm{R}$ & Com & $\mathrm{F}$ \\
\hline 108. & 792 & Crimson breasted Barbet & Megalaima haemacephala & $\mathrm{R}$ & Com & $\mathrm{F}$ \\
\hline \multicolumn{7}{|c|}{ 28. Picidae } \\
\hline 109. & 804 & Rufous Woodpecker & Micropternus brachyurus & $\mathrm{R}$ & L Com & I \\
\hline 110. & 819 & $\begin{array}{l}\text { Lesser golden backed } \\
\text { Woodpecker }\end{array}$ & Dinopium benghalense & $\mathrm{R}$ & L Com & I \\
\hline 111. & 830 & Great black Woodpecker & Dryocopus javensis & $\mathrm{R}$ & L Com & $\mathrm{I}$ \\
\hline 112. & 847 & $\begin{array}{l}\text { Yellow fronted pied } \\
\text { Woodpecker }\end{array}$ & Picoides mahrattensis & $\mathrm{R}$ & L Com & $\mathrm{I}$ \\
\hline 113. & 851 & Pigmy Woodpecker & Picoides nanus & $\mathrm{R}$ & L Com & I \\
\hline
\end{tabular}


G.Y. Dayananda / Our Nature (2009) 7: 100-109

\begin{tabular}{|c|c|c|c|c|c|c|}
\hline \multicolumn{7}{|c|}{ 29. Pittidae } \\
\hline 114. 86 & \multicolumn{2}{|c|}{ Indian Pitta } & Pitta brachyura & \multirow[t]{2}{*}{$\mathrm{R}$} & \multirow[t]{2}{*}{ Com } & \multirow[t]{2}{*}{ I } \\
\hline \multicolumn{4}{|c|}{ 30. Alaudidae } & & & \\
\hline 115. & 873 & Bush Lark & Mirafra assamica & $\mathrm{R}$ & Com & I \\
\hline 116. & 877 & Red winged Bush-Lark & Mirafra erythroptera & $\mathrm{R}$ & L Com & I \\
\hline 117. & 878 & Black bellied Finch-Lark & Eremopterix grisea & RM & L Com & I \\
\hline 118. & 882 & Rufous tailed Finch-Lark & Ammomanes phoenicurus & $\mathrm{R}$ & L Com & I \\
\hline 119. & 901 & Crested Lark & Galerida cristata & $\mathrm{R}$ & L Com & I \\
\hline 120. & 902 & Sykes's Crested Lark & Galerida deva & RM & L Com & $\mathrm{I}$ \\
\hline \multicolumn{7}{|c|}{ 31. Hirundinidae } \\
\hline 121. & 916 & Swallow & Hirundo rustica & RM & L Com & I \\
\hline 122. & 921 & Wire tailed Swallow & Hirundo smithii & RM & L Com & I \\
\hline 123. & 922 & Indian cliff Swallow & Hirundo fluvicola & $\mathrm{R}$ & L Com & I \\
\hline \multicolumn{7}{|c|}{ 32. Laniidae } \\
\hline 124. & 946 & Rufous backed Shrike & Lanius schach & $\mathrm{R}$ & L Com & I \\
\hline \multicolumn{7}{|c|}{ 33. Oriolidae } \\
\hline 125. & 952 & Golden Oriole & Oriolus oriolus & RM & Com & $\mathrm{O}$ \\
\hline 126. & 954 & Black naped Oriole & Oriolus chinensis & $\mathrm{R}$ & Com & $\mathrm{O}$ \\
\hline 127. & 958 & Black headed Oriole & Oriolus xanthornus & $\mathrm{R}$ & Com & $\mathrm{O}$ \\
\hline \multicolumn{7}{|c|}{ 34. Dicruidae } \\
\hline 128. & 963 & King Crow or Black Drongo & Dicrurus adsimilis & $\mathrm{R}$ & Com & I \\
\hline 129 & 965 & Grey or Ashy Drongo & Dicrurus leucophaeus & $\mathrm{R}$ & Com & I \\
\hline 130. & 977 & Racket tailed Drongo & Dicrurus paradiseus & $\mathrm{R}$ & Com & I \\
\hline \multicolumn{7}{|c|}{ 35. Stumidae } \\
\hline 131. & 994 & Brahminy Myna & Sturnuspagodarum & $\mathrm{R}$ & Com & $\mathrm{O}$ \\
\hline 132. & 1006 & Indian Myna & Acridotheres tristis & $\mathrm{R}$ & Com & $\mathrm{O}$ \\
\hline 133. & 1009 & Jungle Myna & Acridotheres fuscus & $\mathrm{R}$ & Com & $\mathrm{O}$ \\
\hline \multicolumn{7}{|c|}{ 36. Corvidae } \\
\hline 134. & 1032 & Tree pie & Dendrocitta vagabunda & $\mathrm{R}$ & Com & $\mathrm{O}$ \\
\hline 135. & 1036 & White bellied Treepie & Dendrocitta leucogastra & $\mathrm{R}$ & Com & $\mathrm{O}$ \\
\hline 136. & 1049 & House Crow & Couvus splendens & $\mathrm{R}$ & V Com & $\mathrm{O}$ \\
\hline 137. & 1054 & Jungle Crow & Corvus macrorhynchos & $\mathrm{R}$ & V Com & $\mathrm{O}$ \\
\hline \multicolumn{7}{|c|}{ 37. Campephagidae } \\
\hline 138. & 1065 & Pied Flycatcher-Shrike & Hemipus picatus & $\mathrm{R}$ & Com & I \\
\hline 139. & 1068 & Large Wood Shrike & Tephrodornis gularis & $\mathrm{R}$ & Com & I \\
\hline 140. & 1070 & Common Wood Shrike & Tephrodornis pandicerianus & $\mathrm{R}$ & V Com & I \\
\hline 141. & 1078 & Black headed cuckoo Shrike & Coracina melanoptera & $\mathrm{R}$ & Com & I \\
\hline 142. & 1081 & Scarlet Minivet & Pericrocotus flammeus & $\mathrm{R}$ & Un Com & I \\
\hline 143. & 1085 & Long tailed Minivet & Pericrocotus ethologus & $\mathrm{R}$ & Un Com & I \\
\hline 144. & 1093 & Small Minivet & Pericrocotus cinnamomeus & $\mathrm{R}$ & Com & I \\
\hline 145. & 1096 & White bellied Minivet & Pericrocotus erythropygius & $\mathrm{R}$ & Com & I \\
\hline
\end{tabular}

38. Irenidae 
G.Y. Dayananda / Our Nature (2009) 7: 100-109

\begin{tabular}{|c|c|c|c|c|c|c|}
\hline 146. & 1098 & Common Iora & Aegithina tiphia & $\mathrm{R}$ & V Com & I \\
\hline 147. & 1102 & Marshall's Iora & Aegithina nigrolutea & $\mathrm{R}$ & Com & I \\
\hline 148. & 1103 & Gold fronted Chloropsis & Chloropsis aurifrons & $\mathrm{R}$ & Com & I \\
\hline \multicolumn{7}{|c|}{ 39. Pycnonotidae } \\
\hline 149. & 1120 & Red whiskered Bulbul & Pycnonotus jocosus & $\mathrm{R}$ & V Com & $\mathrm{F}$ \\
\hline 150. & 1125 & White cheeked Bulbul & Pycnonotus leucogenys & $\mathrm{R}$ & Com & $\mathrm{F}$ \\
\hline 151. & 1128 & Red vented Bulbul & Pycnonotus cafer & $\mathrm{R}$ & V Com & $\mathrm{F}$ \\
\hline 152. & 1148 & Black Bulbul & Hypsipetes madagascariensis & $\mathrm{R}$ & Com & $\mathrm{F}$ \\
\hline
\end{tabular}

40. Muscicapidae

Sub Family :Timalinae

153. 1154 Spotted Babbler

154. 1173 Slaty headed scimitar Babbler

Pellorneum ruficeps

Pomatorhinus horsfieldi

155. 1254 Common Babbler

156. 1258 Large grey Babbler

157. 1265 Jungle Babbler

158. 1267 White headed Babbler

159. $1287 \quad$ Wynaad laughing Thrush

160. 1309 Jerdon's laughing Thrush

161. 1347 White headed shrike Babbler

162. 1396 Black capped Sibia

Sub Family: Muscicapinae

$\begin{array}{lll}\text { 163. } & 1427 & \text { Black and Orange Flycatcher } \\ \text { 164. } & 1442 & \text { Tickll's blue Flycatcher } \\ 165 . & 1445 & \text { Verditer Flycatcher } \\ 166 . & 1446 & \text { Nilgiri verditer Flycatcher } \\ 167 . & 1458 & \text { White spotted fantail Flycatcher } \\ 168 . & 1461 & \text { Paradise Flycatcher } \\ 169 . & 1465 & \text { Black naped blue Flycatcher }\end{array}$

Turdoides caudatus

Turdoides malcolmi

Turdoides striatus

Turdoides affinis

Garrulax delesserti

Garrulax jerdoni

Gampsorhynchus rufulus

Heterophasia capistrata

Muscicapa nigrorufa

Muscicapa tickelliae

Muscicapa thalassina

Muscicapa albicaudata

Rhipidura albicollis

Terpsiphone paradise

Hypothymis azurea

Sub Family: Sylviinae

170. 1506 Rufous fronted Wren-Warbler

171. 1517 Ashy Wren-Warbler

Prinia buchanani

Prinia socialis

Prinia sylvatica

Orthotomus sutorius

173. 1538 Tailor bird

174. 1548 Striated marsh Warbler

175. 1562 Booted Warbler

176. 1575 Common Chiffchaff

177. 1601 Large billed leaf Warbler

Sub Family: Turdinae

$\begin{array}{lll}\text { 178. } & 1650 & \text { Blue Chat } \\ \text { 179. } & 1661 & \text { Magpie Robin } \\ 180 . & 1665 & \text { Shama } \\ 181 . & 1692 & \text { Brown rock Chat } \\ 182 . & 1700 & \text { Pied bush Chat }\end{array}$

Megalurus palustris

Hippolais caligata

Phylloscopus collybita

Phylloscopus occipitalis

Erithacus brunneus

Copsychus saularis

Copsychus malabaricus

Cercomela fusca

Saxicola caprata

$\begin{array}{lll}\mathrm{R} & \text { Com } & \mathrm{I} \\ \mathrm{R} & \text { Com } & \mathrm{I} \\ \mathrm{R} & \text { V Com } & \mathrm{I} \\ \mathrm{R} & \text { V Com } & \mathrm{I} \\ \mathrm{R} & \text { V Com } & \mathrm{I} \\ \mathrm{R} & \text { Com } & \mathrm{I} \\ \mathrm{R} & \text { Un Com } & \mathrm{I} \\ \mathrm{R} & \text { Un Com } & \mathrm{I} \\ \mathrm{R} & \text { Un Com } & \mathrm{I} \\ \mathrm{R} & \text { Com } & \mathrm{I}\end{array}$

$\begin{array}{lll}\text { RM } & \text { Un Com } & \text { I } \\ \text { R } & \text { Com } & \text { I } \\ \text { R } & \text { Com } & \text { I } \\ \text { R } & \text { Com } & \text { I } \\ \text { R } & \text { Com } & \text { I } \\ \text { R } & \text { Com } & \text { I } \\ \text { R } & \text { Com } & \text { I }\end{array}$

RM Com I

M $\quad \mathrm{Ra} \quad \mathrm{I}$

M $\quad \mathrm{Ra} \quad \mathrm{I}$

RM V Com I

$\mathrm{R}$ Un Com I

$\mathrm{R}$ Un Com I

$\mathrm{R}$ Un Com I

$\mathrm{R}$ Un Com I

R V Com I

RM V Com I

R Un Com I

RM Un Com I

R Com I 
G.Y. Dayananda / Our Nature (2009) 7: 100-109

\begin{tabular}{|c|c|c|c|c|c|c|}
\hline 183. & 1704 & Jerdon's bush Chat & Saxicola jerdoni & $\mathrm{R}$ & Com & $\mathrm{I}$ \\
\hline 184. & 1720 & Indian Robin & Saxicoloides fulicata & $\mathrm{R}$ & V Com & $\mathrm{I}$ \\
\hline 185. & 1726 & Blue Rock Thrush & Monticola solitarius & $\mathrm{R}$ & Com & I \\
\hline 186. & 1733 & Orange headed Thrush & Zoothera citrine cyanotus & $\mathrm{R}$ & Com & I \\
\hline 187. & 1751 & Black Bird & Turdus merula & $\mathrm{RM}$ & Com & I \\
\hline \multicolumn{7}{|c|}{ 41. Paridae } \\
\hline 188. & 1794 & Grey Tit & Parus major & RM & Com & I \\
\hline 189. & 1798 & White winged Black Tit & Parus nuchalis & $\mathrm{R}$ & Com & $\mathrm{I}$ \\
\hline 190. & 1810 & Yellow cheeked Tit & Parus xanthogenys & $\mathrm{R}$ & Com & $\mathrm{I}$ \\
\hline \multicolumn{7}{|c|}{ 42. Sittidae } \\
\hline 191. & 1830 & Chestnut bellied Nuthatch & Sitta castanea & $\mathrm{R}$ & Com & $\mathrm{I}$ \\
\hline \multicolumn{7}{|c|}{ 43. Motacillidae } \\
\hline 192. & 1859 & Paddy field Pipit & Anthus rufulus & $\mathrm{R}$ & L Com & I \\
\hline 193. & 1874 & Forest Wagtail & Motacilla indica & $\mathrm{R}$ & L Com & I \\
\hline 194. & 1876 & Yellow Wagtail & Motacilla flava & $\mathrm{R}$ & Lcom & I \\
\hline 195. & 1885 & White Wagtail & Motacilla alba & $\mathrm{R}$ & Com & I \\
\hline 196. & 1891 & Large pied Wagtail & Motacilla maderaspatensis & $\mathrm{R}$ & Lcom & I \\
\hline \multicolumn{7}{|c|}{ 44. Dicaeidae } \\
\hline 197. & 1899 & Tickell's Flowerpecker & Dicaeum erythrorhynchos & $\mathrm{R}$ & L Com & $\mathrm{F}, \mathrm{N}, \mathrm{I}$ \\
\hline 198. & 1902 & Plain coloured Flowerpecker & Dicaeun concolor & $\mathrm{R}$ & L Com & $\mathrm{F}, \mathrm{N}, \mathrm{I}$ \\
\hline 199. & 1905 & Fire breasted Flowerpecker & Dicaeun ignipectus & $\mathrm{R}$ & L Com & $\mathrm{F}, \mathrm{N}, \mathrm{I}$ \\
\hline \multicolumn{7}{|c|}{ 45. Nectariniidae } \\
\hline 200. & 1908 & Puple rumped Sunbird & Nectarinia zeylonica & $\mathrm{R}$ & Com & $\mathrm{F}, \mathrm{N}, \mathrm{I}$ \\
\hline 201. & 1909 & Small Sunbird & Nectarinia minima & $\mathrm{R}$ & Com & $\mathrm{F}, \mathrm{N}, \mathrm{I}$ \\
\hline 202. & 1912 & Maroon breasted Sunbird & Nectarinia lotenia & $\mathrm{R}$ & Com & $\mathrm{F}, \mathrm{N}, \mathrm{I}$ \\
\hline 203. & 1917 & Purple Sunbird & Nectarinia asiatica & $\mathrm{R}$ & Com & $\mathrm{F}, \mathrm{N}, \mathrm{I}$ \\
\hline 204. & 1931 & Little Spider hunter & Arachnothera longirostra & $\mathrm{R}$ & L Com & I \\
\hline \multicolumn{7}{|c|}{ 46. Zosteropidae } \\
\hline 205. & 1933 & White Eye & Zosterops palpebrosus & $\mathrm{RM}$ & L Com & $\mathrm{F}, \mathrm{N}$ \\
\hline \multicolumn{7}{|c|}{ 47. Ploceidae } \\
\hline \multicolumn{7}{|c|}{ Sub Family : Passerinae } \\
\hline 206. & 1938 & House Sparrow & Passer domesticus & $\mathrm{R}$ & Com & $\mathrm{G}, \mathrm{I}$ \\
\hline \multicolumn{7}{|c|}{ Sub Family: Ploceinae } \\
\hline 207. & 1957 & Baya weaverbird & Ploceus philippinus & $\mathrm{R}$ & Com & G \\
\hline 208. & 1961 & Balck breasted weaver bird & Ploceus benghalensis & $\mathrm{R}$ & Com & $\mathrm{G}$ \\
\hline 209. & 1962 & Streaked weaverbird & Ploceus manyar & $\mathrm{R}$ & L Com & G \\
\hline \multicolumn{7}{|c|}{ Sub Family: Estrildinae } \\
\hline 210. & 1964 & Red Munia & Estrilda amandava & $\mathrm{R}$ & Com & G \\
\hline 211. & 1965 & Green Munia & Estrilda formosa & $\mathrm{R}$ & Un Com & G \\
\hline 212. & 1966 & White throated Munia & Lonchura malabarica & $\mathrm{R}$ & Com & G \\
\hline 213. & 1968 & White backed Munia & Lonchura striata & $\mathrm{R}$ & Com & G \\
\hline 214. & 1974 & Spotted Munia & Lonchura punctulata & $\mathrm{R}$ & Com & $\mathrm{G}$ \\
\hline 215. & 1978 & Black headed Munia & Lonchura malacca & $\mathrm{R}$ & Com & G \\
\hline
\end{tabular}


G.Y. Dayananda / Our Nature (2009) 7: 100-109

\begin{tabular}{|c|c|c|c|c|c|c|}
\hline \multicolumn{7}{|c|}{ 48. Fringillidae } \\
\hline \multicolumn{7}{|c|}{ Sub Family: Carduelinae } \\
\hline 216. & 2013 & Rose Finch & Carpodacus erythrinus & $\mathrm{RM}$ & Com & $\mathrm{F}, \mathrm{G}, \mathrm{N}$ \\
\hline \multicolumn{7}{|c|}{ Sub Family: Emberizinae } \\
\hline 217. & 2057 & House Striolated & Emberiza striolata & $\mathrm{R}$ & L Com & $\mathrm{G}$ \\
\hline 218. & 2060 & Crested Bunting & Emberiza lathami & $\mathrm{R}$ & L Com & $\mathrm{G}$ \\
\hline
\end{tabular}

Synopsis numbers as followed by Pittie and Robertson (1993), Ali and Ripley (1983) and Ali (1996); Common and scientific names by Manakad an and Pittie (2001) and Abundance Status by Kumar et al. (2003).

Food habit of the birds: $\mathrm{I}=$ Insectivore, $\mathrm{P}=$ Piscivore, $\mathrm{C}=$ Carnivore, $\mathrm{N}=$ Nectivore, $\mathrm{F}=$ Frugivore, $\mathrm{O}=$ Omnivore, $\mathrm{G}=$ Granivore, $\mathrm{I} / \mathrm{G}=$ Insectivore with Grainivore

Status of the birds: $\mathrm{WM}=\mathrm{W}$ inter migrant, $\mathrm{Com}=\mathrm{Common}, \mathrm{R} / \mathrm{LM}=$ Resident with local movements, $\mathrm{L}$ Com= Locally common, $\mathrm{R}=$ Resident, Un Com= Un common, $\mathrm{M}=$ Migratory, $\mathrm{V}$ Com= Very co mmon, $\mathrm{R} / \mathrm{LM} / \mathrm{SM}=$ Resident with local as well as summer movements. $\mathrm{RS}^{1}=$ Residential status, $\mathrm{AS}^{2}=$ Abundance status, $\mathrm{FH}^{3}=$ Food habit. 